\title{
1-Jahres-Ergebnisse nach kombinierter Kataraktoperation und Excimer-Laser-Trabekulotomie bei erhöhtem Intraokulardruck
}

Töteberg-Harms, M ; Ciechanowski, P P ; Hirn, C ; Funk, J

DOI: https://doi.org/10.1007/s00347-011-2337-6

Other titles: One-year results after combined cataract surgery and excimer laser trabeculotomy for elevated intraocular pressure

Posted at the Zurich Open Repository and Archive, University of Zurich ZORA URL: https://doi.org/10.5167/uzh-47539

Journal Article

Published Version

Originally published at:

Töteberg-Harms, M; Ciechanowski, P P; Hirn, C; Funk, J (2011). 1-Jahres-Ergebnisse nach kombinierter Kataraktoperation und Excimer-Laser-Trabekulotomie bei erhöhtem Intraokulardruck. Der Ophthalmologe, 108(8):733-738.

DOI: https://doi.org/10.1007/s00347-011-2337-6 
Ophthalmologe $2011 \cdot 108: 733-738$

DOI 10.1007/s00347-011-2337-6

Online publiziert: 27. Februar 2011

(c) Springer-Verlag 2011

\author{
M. Töteberg-Harms · P.P. Ciechanowski · C. Hirn · J. Funk \\ UniversitätsSpital Zürich, Augenklinik, Zürich
}

\title{
1-Jahres-Ergebnisse nach kombinierter Kataraktoperation und Excimer-Laser-Trabekulotomie bei erhöhtem Intraokulardruck
}

\section{Hintergrund und Fragestellung}

Das Glaukom ist eine weitverbreitete $\mathrm{Au}$ generkrankung, die unbehandelt zu Sehbeeinträchtigung führt. Sie ist eine der 3 häufigsten Gründe für Blindheit weltweit $[36,45]$ und nach wie vor der häufigste Grund für Blindheit in Industrieländern $[33,35,44]$. Das Glaukom ist eine chronisch progressive neurodegenerative Erkrankung, die zu einem Verlust von Ganglienzellen und Axonen führt [34]. Charakteristisch sind eine vergrößerte Exkavation der Papille und Gesichtsfeldausfälle. In den meisten Fällen ist der Intraokulardruck (IOD) über die Norm erhöht.

Der IOD ist der wichtigste Risikofaktor für ein Glaukom. Bei der Behandlung des Glaukoms ist die Senkung des IOD die einzige evidenzbasierte Therapie $[8,9$, 13, 14, 30, 43]. In den meisten Fällen ist die medikamentöse Senkung des IOD zu Beginn Therapie der Wahl [11, 14, 53]. Wenn dies nicht mehr ausreichend ist, gibt es zahlreiche etablierte Operationsmethoden zur Senkung des IOD. Goldstandard ist immer noch die Trabekulektomie (TE).

Die TE, so wie sie heute durchgeführt wird, wurde erstmals 1968 von Cairns beschrieben [7]. Hierbei wird Kammerwasser durch einen präparierten Skleradeckel aus der Vorderkammer in den Subkonjunktivalraum geleitet [41]. Die TE ist eine sehr effektive Operationsmethode, um den IOD langfristig zu senken [20, 25]. Alle Operationsmethoden, TE ebenso wie z. B. Laseroperationen (Argon- oder selektive Laser-Trabekuloplastik, Zyklophotokoagulation), haben jedoch zum Teil erhebliche Nebenwirkungen.
Der Hauptgrund für einen erhöhten IOD ist ein verminderter Kammerwasserabfluss [4, 12, 18, 21, 31, 32, 37, 46, 47], während die Kammerwasserproduktion annähernd konstant bleibt $[3,5,6]$. Daher scheint es sinnvoll, operativ den Kammerwasserabfluss zu verbessern. Die ExcimerLaser-Trabekulotomie (ELT) ab interno ist eine minimal-invasive Operationsmethode, um den IOD bei Patienten mit Glaukom oder okulärer Hypertension zu senken. Hierbei werden Poren von der Vorderkammer in den Schlemm-Kanal angelegt $[1,2,15,19,22,27,29,42,48,49-52$, 54]. Eine ELT kann ohne größeren Aufwand am Ende einer Clear-cornea-Phakoemulsifikation erfolgen. Die ELT kann den IOD für eine lange Zeit mit einer geringen Komplikationsrate senken [2, 15, 29, 48, 54]. Die Nebenwirkungen sind weitaus geringer, und die Rekonvaleszenzzeit ist deutlich kürzer als bei einer TE.

Das Ziel dieser Studie war es, den Erfolg der IOD-Senkung durch eine kombinierte Katarakt- und Glaukomoperation (Phakoemulsifikation mit Hinterkammerlinsenimplantation und Excimer-Laser-Trabekulotomie, Phako-ELT) nach 12 Monaten an Patienten mit einem präoperativen IOD von $>21 \mathrm{mmHg}$ mit Glaukom oder okulärer Hypertension zu untersuchen.

\section{Studiendesign und Untersuchungsmethoden}

Es wurden 24 Augen von 24 konsekutiven Patienten 12 Monate \pm 2 Wochen nach Phako-ELT nachuntersucht. Alle Patienten wurden in unserer Poliklinik nachkontrolliert. Der IOD wurde mittels Goldmann-Applanationstonometrie gemessen, der bestkorrigierte Visus wurde bestimmt (BCVA, „snellen charts“), eine Spaltlampenuntersuchung von Vorder- und Hinterabschnitt erfolgte, und die Glaukommedikation wurde erhoben. Alle Untersuchungen erfolgten durch einen Untersucher (M.T.-H.). Indikationen für die Phako-ELT waren eine Katarakt und ein moderat erhöhter IOD über $21 \mathrm{mmHg}$ ohne medikamentöse Therapie oder eine moderate Katarakt (BCVA $\leq 0,8$ Snellen) und ein IOD größer als $21 \mathrm{mmHg}$ unter medikamentöser Therapie.

Einschlusskriterien waren eine okuläre Hypertension oder ein manifestes Glaukom mit einem IOD über $21 \mathrm{mmHg}$ und typisch glaukomatös vergrößerter Exkavation der Papille oder typischen Gesichtsfeldausfällen oder beidem. Zusätzlich musste der Kammerwinkel offen sein (Grad III oder IV nach Shaffer). Patienten mit weit fortgeschrittenem Glaukom (d. h. fixationsbedrohende Gesichtsfelddefekte) oder mit einem IOD $\geq 35 \mathrm{mmHg}$ wurden ausgeschlossen. Ebenso wurden Patienten mit anderen Neuropathien des Sehnerven außer Glaukom ausgeschlossen. Primäre Studienendpunkte waren IOD und Anzahl an antiglaukomatösen Substanzen. Sekundäre Studienendpunkte waren BCVA, intra- und postoperative Komplikationen sowie nötige Glaukomfolgeoperationen.

\section{Operationstechnik}

Alle Operationen wurden durch einen Operateur (J.F.) durchgeführt. Eine Stan- 
Tab. 1 Demografische Daten

\begin{tabular}{|ll} 
Studienaugen & $\begin{array}{l}28 \text { Augen von 28 Patienten } \\
\text { (13 rechte, 15 linke Augen) } \\
\text { (4 Augen exkludiert wegen Reoperation) }\end{array}$ \\
\hline Durchschnittsalter & $74,33 \pm 11,81$ \\
\hline Geschlecht & 10 Männer $(35,71 \%), 18$ Frauen $(64,29 \%)$ \\
\hline Diagnosen & \\
Primäres Offenwinkelglaukom & $9(32,14 \%)$ \\
\hline Pseudoexfoliationsglaukom & $15(53,57 \%)$ \\
\hline Okuläre Hypertension & $3(10,71 \%)$ \\
\hline Sekundärglaukom (nach Contusio bulbi) & $1(3,57 \%)$ \\
\hline
\end{tabular}

Tab. 2 Statistische Daten von BCVA, IOD und AGD vor und nach Phako-ELT

\begin{tabular}{|c|c|c|c|c|c|c|c|c|c|c|c|}
\hline \multicolumn{4}{|l|}{ BCVA } & \multicolumn{4}{|l|}{$I O D$} & \multicolumn{4}{|l|}{ AGD } \\
\hline Prä & & Post & & Prä & & Post & & Prä & & Post & \\
\hline $\begin{array}{l}\text { Mean } \\
\pm \text { SD }\end{array}$ & $\begin{array}{l}95 \%- \\
\mathrm{KI}\end{array}$ & $\begin{array}{l}\text { Mean } \\
\pm \text { SD }\end{array}$ & $\begin{array}{l}95 \%- \\
\text { KI }\end{array}$ & $\begin{array}{l}\text { Mean } \\
\pm \text { SD }\end{array}$ & 95\%-KI & $\begin{array}{l}\text { Mean } \\
\pm \text { SD }\end{array}$ & 95\%-KI & $\begin{array}{l}\text { Mean } \\
\pm \text { SD }\end{array}$ & $\begin{array}{l}95 \%- \\
\text { KI }\end{array}$ & $\begin{array}{l}\text { Mean } \\
\pm \text { SD }\end{array}$ & $\begin{array}{l}95 \%- \\
\mathrm{KI}\end{array}$ \\
\hline $\begin{array}{l}0,45 \\
\pm 0,25\end{array}$ & $\begin{array}{l}0,34- \\
0,55\end{array}$ & $\begin{array}{l}0,78 \\
\pm 0,30\end{array}$ & $\begin{array}{l}0,65- \\
0,91\end{array}$ & $\begin{array}{l}25,33 \\
\pm 2,85\end{array}$ & $\begin{array}{l}24,13- \\
26,54\end{array}$ & $\begin{array}{l}16,54 \\
\pm 4,95\end{array}$ & $\begin{array}{l}14,45- \\
18,63\end{array}$ & $\begin{array}{l}2,25 \\
\pm 1,26\end{array}$ & $\begin{array}{l}1,72- \\
2,78\end{array}$ & $\begin{array}{l}1,46 \\
\pm 1,38\end{array}$ & $\begin{array}{l}0,87- \\
2,04\end{array}$ \\
\hline
\end{tabular}

Prä vor Phako-ELT, Post 12 Monate nach Phako-ELT, SD Standardabweichung, KI Konfidenzintervall.

Tab. 3 Reduktion von IOD und AGD vor und nach Phako-ELT

\begin{tabular}{|lllll}
\hline & Mittelwert \pm SD & $(\%)$ & Konfidenzintervall & p-Wert \\
$\Delta \mathrm{IOD}$ (prä-post) & $8,79 \pm 5,28 \mathrm{mmHg}$ & $-37,70$ & $6,56-11,02$ & $\mathrm{p}<0,001$ \\
$\Delta \mathrm{AGD}$ (prä-post) & $0,79 \pm 1,50$ & $-62,70$ & $0,16-1,43$ & $\mathrm{p}=0,017$ \\
\hline prä vor Phako-ELT, post 12 Monate nach Phako-ELT, SD Standardabweichung. & \\
\hline
\end{tabular}

dard-clear-cornea-Phakoemulsifikation und intrakapsuläre Linsenimplantation (Alcon MA 50 BM, Alcon Inc., Hünenberg, Schweiz) erfolgten zuerst, anschließend eine medikamentöse Miose mit Acetylcholinchlorid (Miochol ${ }^{\circ}$, Novartis Pharma Schweiz AG, Bern, Schweiz). Die Vorderkammer wurde mit Viskoelastikum (Natriumhyaluronat, Healon) vertieft. Ein endoskopisch kontrollierter photoablativer Laser (Excimer laser, AIDA, TUI-Laser, München, Deutschland, Pulsenergie $1,2 \mathrm{~mJ}$, Pulsdauer $60 \mathrm{~ns}$ ), der bei einer Wellenlänge von $308 \mathrm{~nm}$ arbeitet, wurde benutzt, um 1o Mikroperforationen im Trabekelwerk über einen Winkel von $90^{\circ}$ zu erzeugen. Jede Perforation hat einen ungefähren Durchmesser von $0,5 \mathrm{~mm}$. Um die Energie verlustfrei zu übertragen, muss die Laserfaser in Kontakt zum Trabekelwerk sein. Nach der Applikation der Laserenergie kann eine Luftblasenbildung zusammen mit einer kleinen retrograden Blutung aus dem Schlemm-Kanal beobachtet werden. Dies zeigt an, dass eine erfolgreiche Perforation von Trabekelmaschenwerk und der inneren Wand des Schlemm-Kanals ent- standen ist. Die Blutung sistiert spontan, wenn am Ende der Operation das Viskoelastikum aus der Vorderkammer gespült, der Bulbus auf ungefähr $15 \mathrm{mmHg}$ tonisiert und Tunnel und Parazenthesen verquollen sind.

\section{Statistik}

Deskriptive Statistik für quantitative Variablen wie Mittelwert, Standardabweichung, 95\%-Konfidenzintervall und relative Häufigkeit von qualitativen Variablen wurde für alle Studienaugen (ohne „dropouts“) berechnet. Ergebnisse sind als arithmetisches Mittel \pm Standardabweichung angegeben. Der „students t test“ wurde benutzt, um signifikante Änderungen bei IOD und Anzahl der antiglaukomatösen Medikamente (AGD) zu bestimmen. Als Signifikanzniveau ist $\mathrm{p}<0,05 \mathrm{de}-$ finiert. Weiterhin wurden die Anzahl der Patienten, die die Kriterien von Erfolg erfüllen, und deren relative Häufigkeit gezählt. Eine Behandlung wurde als erfolgreich definiert, wenn der postoperative IOD kleiner als $21 \mathrm{mmHg}$ lag und die IOD-Reduktion mindestens $20 \%$ be- trug ohne medikamentöse Therapie bzw. mit medikamentöser Therapie („qualified success"). Eine Folgeoperation aufgrund ungenügender Drucksenkung wurde als Misserfolg gewertet. Statistische Analysen erfolgten mit Microsoft Excel Version 2008 und SPSS/PASW Statistics Version 18.

Alle Studienteilnehmer gaben ihr schriftliches Einverständnis. Die Studie ist geprüft und bewilligt von der zuständigen kantonalen Ethikkommission (KEK-ZHNr. 881, 06/07/2009) und in Übereinstimmung mit der Deklaration von Helsinki und den gültigen kantonalen und nationalen Gesetzen.

Die Ergebnisse der Studie wurden vorab als Vortrag am World Ophthalmology Congress 2010 im Rahmen des 108. Kongress der Deutschen Ophthalmologischen Gesellschaft in Berlin, Deutschland, präsentiert.

\section{Ergebnisse}

In die Studie wurden 28 Augen von $28 \mathrm{~Pa}$ tienten mit einem Durchschnittsalter von $74,33 \pm 11,81$ Jahren eingeschlossen. Die demografischen Daten sind in • Tab. 1 wiedergegeben. Vier Patienten benötigten im Nachbeobachtungszeitraum von $12 \mathrm{Mo-}$ naten Folgeoperationen, um den IOD zu kontrollieren ( 2 erhielten eine TE, 2 eine Zyklophotokoagulation). Diese wurden als „dropouts“ klassifiziert und gingen als Behandlungsfehler in die Kalkulation der Erfolgsrate ein. Sie wurden nicht in die Berechung des durchschnittlichen IOD und der IOD-Änderung mit einbezogen, um die IOD-Senkung durch PhakoELT nicht durch die Folgeoperation (insbesondere durch die TE) in Richtung zu positiveren Ergebnissen zu verfälschen. In die Statistik gingen - mit Ausnahme der Erfolgsraten - daher nur 24 Augen von 24 Patienten ein. Nur wenige Patienten zeigten einen leichten Vorderkammerreiz nach der Operation, so wie er gewöhnlich nach Kataraktoperation gesehen wird. Es gab keine schwerwiegenden Operationskomplikationen, insbesondere keine Endophthalmitis oder ausgeprägte Fibrinreaktion.

Präoperativ betrug der BCVA

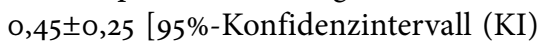
0,34-0,55], der IOD war 25,33 $\pm 2,85 \mathrm{mmHg}$ 


\section{Töteberg-Harms · P.P. Ciechanowski · C. Hirn · J. Funk \\ 1-Jahres-Ergebnisse nach kombinierter Kataraktoperation und Excimer-Laser-Trabekulotomie bei erhöhtem Intraokulardruck}

\section{Zusammenfassung}

Hintergrund. Glaukom ist eine der häufigsten Ursachen für Blindheit. Normalerweise ist ein erhöhter Abflusswiderstand die Ursache, während die Kammerwasserproduktion normal bleibt. Medikamentöse Drucksenkung ist in der Regel die First-line-Behandlung. Der operative Goldstandard ist die Trabekulektomie (TE). Die TE hat jedoch zahlreiche postoperative Komplikationen. Deswegen bevorzugen wir für ausgesuchte Glaukompatienten die kombinierte Kataraktoperation mit der Excimer-Laser-Trabekulotomie (Phako-ELT). Indikationen sind eine Katarakt und gleichzeitig ein moderat erhöhter Intraokulardruck (IOD) ohne medikamentöse Therapie oder eine moderate Katarakt mit erhöhtem IOD trotz medikamentöser Therapie. Patienten und Methoden. Im Rahmen der ELT werden 10 Poren über einen Winkel von $90^{\circ} \mathrm{im}$ Trabekelmaschenwerk erzeugt. Es wurden 28 Augen von 28 Patienten
(10 Männer, 18 Frauen) 12 Monate \pm 2 Wochen nach kombinierter Phako-ELT nachuntersucht. Vier Patienten wurden wegen einer drucksenkenden Reoperation während des Nachbeobachtungszeitraums ausgeschlossen. Intraokulardruck, bestkorrigierter Visus und Anzahl der Glaukommedikamente („,anti-glaucoma drugs“, AGD) wurden erhoben und eine Spaltlampenuntersuchung durchgeführt.

Ergebnisse. Das Durchschnittsalter lag bei $74,33 \pm 11,81$ Jahren. Diagnosen waren primäres Offenwinkelglaukom bei 9 Augen, Pseudoexfoliationsglaukom bei 15 Augen, okuläre Hypertension und posttraumatisches Sekundärglaukom bei 3 bzw. 1 Auge. Im Durchschnitt konnte durch Phako-ELT der IOD um 8,79 $\pm 5,28 \mathrm{mmHg}(-34,70 \%$, $p<0,001)$ gesenkt werden. Die Anzahl der AGD konnte gleichzeitig um $0,79 \pm 1,50$ $(-62,70 \%, p=0,017)$ reduziert werden.

\section{One-year results after combined cataract surgery and excimer laser trabeculotomy for elevated intraocular pressure}

\section{Abstract}

Background. Glaucoma is one of the most common reasons for blindness. Usually an elevated resistance to aqueous outflow is the reason, while aqueous humor production is still normal. Medical reduction of intraocular pressure (IOP) is the first-line therapy in most cases. The gold standard of surgical treatment is trabeculectomy (TE). But TE has a lot of postoperative complications. Therefore we prefer the combined procedure of cataract extraction plus excimer laser trabeculotomy (phaco-ELT) for a selected group of glaucoma patients. Indications are cataract together with moderately elevated IOP without medical therapy or a moderate cataract together with elevated IOP under medical therapy. Patients and Methods. During ELT, 10 pores were created over $90^{\circ}$ of the anterior chamber angle; 28 eyes of 28 patients ( 10 men and
18 women) were reexamined 12 months \pm 2 weeks after combined phaco-ELT. Four patients were excluded because of IOP-lowering surgery during the follow-up. IOP, best corrected visual acuity, slit lamp biomicroscopy as well as glaucoma medication history (antiglaucoma drugs, AGD) were recorded. Results. The mean age was $74.33 \pm 11.81$ years. The diagnosis was primary open-angle glaucoma in 9 eyes, pseudoexfoliative glaucoma in 15 eyes, ocular hypertension in 3 eyes, and 1 post-traumatic secondary glaucoma. On average, phaco-ELT could reduce the IOP by $8.79 \pm 5.28 \mathrm{mmHg}(-34.70 \%, p<0.001)$. AGD could be reduced by $0.79 \pm 1.50$ $(-62.70 \%, p=0.017)$ at the same time. Conclusion. The ELT is easy to perform at the end of cataract surgery. Duration of surgery is only prolonged by 2 to 3 minutes.
Schlussfolgerungen. Eine ELT ist technisch einfach am Ende einer Kataraktoperation durchzuführen. Die Operationszeit wird lediglich um 2-3 min verlängert. Wir fanden eine durchschnittliche IOD-Reduktion von $8,79 \mathrm{mmHg}(-34,70 \%)$ und gleichzeitig eine durchschnittliche Reduktion von AGD um 0,79 . Es ist bekannt, dass der Effekt der IODReduktion konstant mit der Zeit ist im Gegensatz zur Argon- oder selektiven Laser-Trabekuloplastik. Eine evtl. spätere fistulierende Operation ist nicht beeinträchtigt, da die Bindehaut nicht berührt wird und es folglich keine Vernarbung gibt. Für ausgewählte Glaukompatienten ist diese Operation eine Möglichkeit, die Trabekulektomie zu vermeiden.

\section{Schlüsselwörter}

Glaukom · Excimer-Laser-Trabekulotomie . Trabekelmaschenwerk - Phakoemulsifikation . Laseroperation
We found an average IOP reduction of 8.79 $\mathrm{mmHg}(-34.70 \%)$ and an average reduction of 0.79 AGD. It is known that the effect of IOP reduction is constant over time unlike argon or selective laser trabeculoplasty. If needed later on, filtering surgery is not compromised because there is no conjunctival touch during ELT and therefore no scarring of the conjunctiva. For a selected collective of glaucoma patients this procedure could be a good way to avoid trabeculectomy.

\section{Keywords}

Glaucoma - Excimer laser trabeculotomy . Trabecular meshwork - Phacoemulsification . Laser surgery 


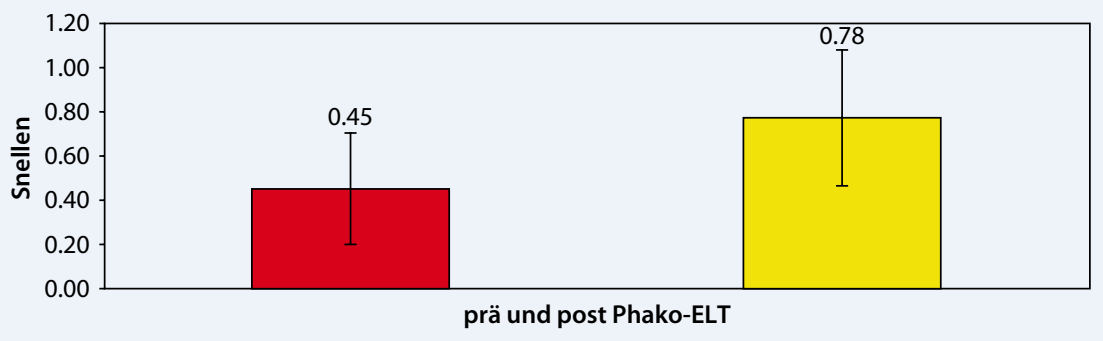

Abb. $1 \Delta$ Visus (BCVA) vor Phako-ELT und am Ende des Nachbeobachtungszeitraums von 12 Monaten (angegeben sind der Mittelwert und die Standardabweichung als Fehlerbalken)

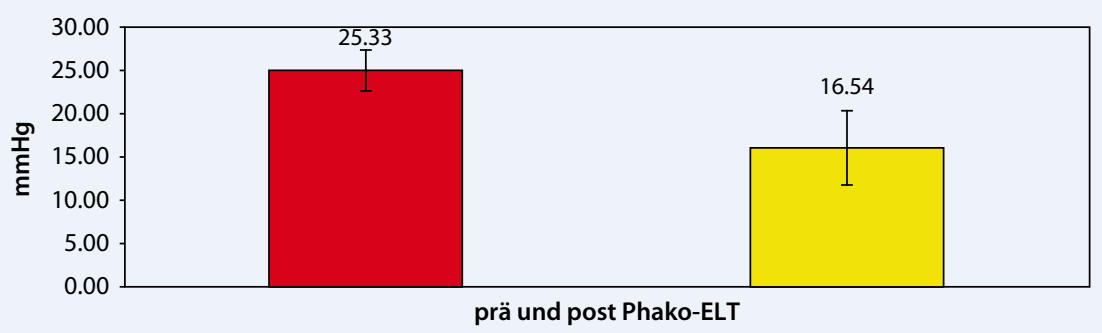

Abb. $2 \Delta$ IOD vor Phako-ELT und am Ende des Nachbeobachtungszeitraums von 12 Monaten (angegeben sind der Mittelwert und die Standardabweichung als Fehlerbalken)

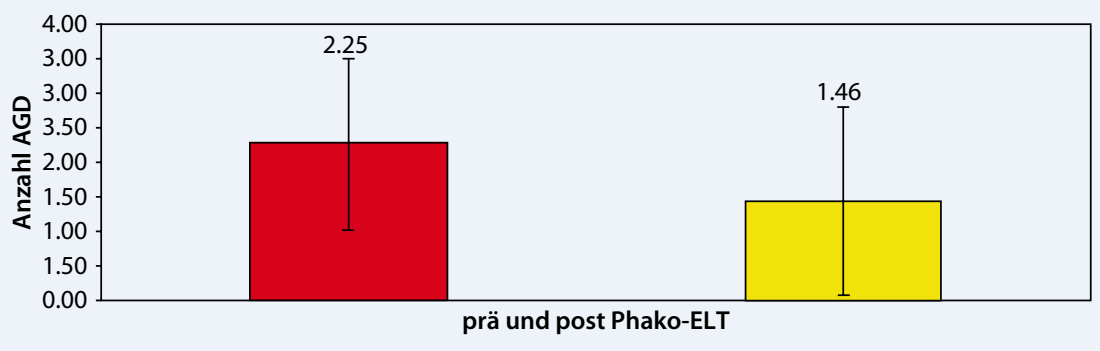

Abb. $3 \Delta$ Anzahl antiglaukomatöser Medikamente vor Phako-ELT und am Ende des Nachbeobachtungszeitraums von 12 Monaten (angegeben sind der Mittelwert und die Standardabweichung als Fehlerbalken)

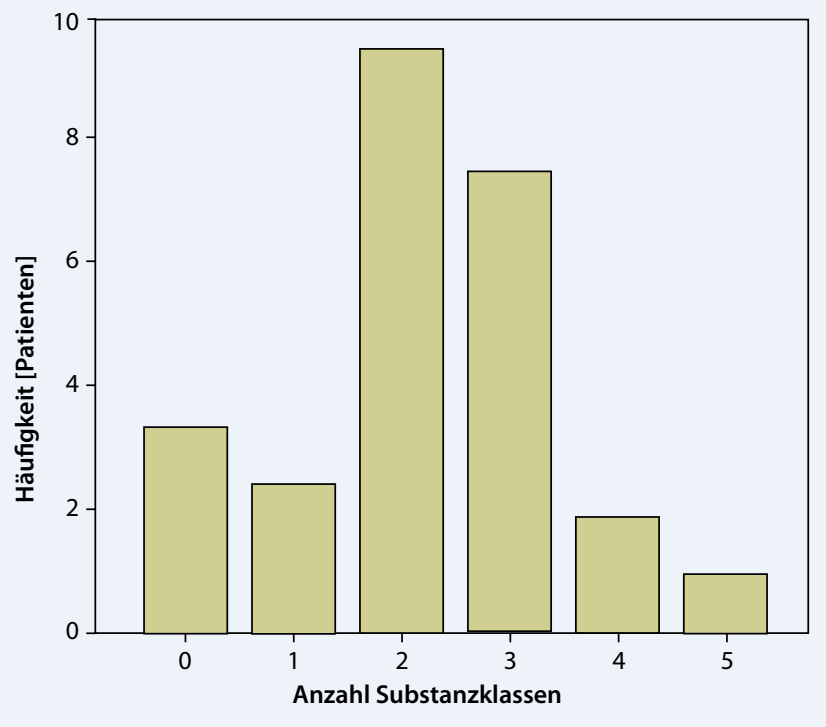

Abb. $4<$ Häufigkeit der applizierten drucksenkenden Medikamente präoperativ: Anzahl Patienten über Anzahl Substanzklassen drucksenkende Medikamente $(0=$ keine, $1=1$ Substanzklasse, $\ldots, 5=4$ lokale plus eine systemische Substanzklasse)
(95\%-KI 24,13-26,54), und es wurden im Mittel 2,25 $\pm 1,26$ AGD (95\%-KI 1,722,78) appliziert. Am Ende des Nachbeobachtungszeitraums nach Phako-ELT lag der BCVA bei o,78 $\pm 0,30$ (95\%-KI o,65$0,91)$, der IOD lag bei $16,54 \pm 4,95 \mathrm{mmHg}$ (95\%-KI 14,45-18,63), und im Mittel wurden noch 1,46 $\pm 1,38$ AGD (95\%-KI o,872,04) appliziert. Vergleicht man IOD und AGD vor und nach Phako-ELT, so findet sich eine mittlere IOD-Senkung von $8,79 \pm 5,28 \mathrm{mmHg}(-34,70 \%$, 95\%-KI 6,56$11,02, \mathrm{p}<0,001)$ und eine Reduktion der AGD von $0,79 \pm 1,50(-62,70 \%, 95 \%-\mathrm{KI}$ $0,16-1,43, p=0,017)$. Alle Ergebnisse sind in - Tab. 2 und in den $\bullet$ Abb. 1-5 die Änderungen von IOD und AGD von vor zu nach Phako-ELT in $\bullet$ Tab. 3 wiedergegeben.

Am Ende des Nachbeobachtungszeitraumes von 12 Monaten erfüllten 6 von 28 Augen alle Erfolgskriterien (IOD $<22 \mathrm{mmHg}$ plus IOD-Reduktion von mindestens $20 \%$ und keine AGD) und 18 von 28 Augen $(64,3 \%)$ einen "qualified success".

\section{Diskussion}

Die Excimer-Laser-Trabekulotomie ist ein einfacher komplikationsarmer Eingriff, der problemlos am Ende einer Clear-cornea-Kataraktoperation durchgeführt werden kann. Die Operationszeit verlängert sich lediglich um ca. 2-3 min. Es kann der bereits präparierte korneale Tunnel der Kataraktoperation als Zugang benutzt werden.

Bei einer Wellenlänge von $308 \mathrm{~nm}$ wird das Trabekelmaschenwerk schonend abladiert. Es entstehen Mikroperforationen zwischen Vorderkammer und Schlemm-Kanal $[16,28,49]$. Es gibt praktisch keine thermischen Nebeneffekte und keine Schäden an der Außenwand des Schlemm-Kanals.

Die punktuelle Ablation des Trabekelmaschenwerks durch einen Prototyp eines Excimer-Lasers mit Kontaktglas wurde erstmalig 1996 von Vogel et al. [49] beschrieben. Die ELT-Technik unterscheidet sich von anderen minimal-invasiven Lasertechniken in der Glaukomchirurgie wie ALT oder SLT. ALT erzeugt Gewebeveränderungen durch Hitze, und SLT erzeugt „Geweberemodeling“, weswegen 
der Effekt mit der Zeit nachlässt. Nach ELT zeigen sich in elektronenmikroskopischen Untersuchungen sehr glatte Kanten der Poren, was die Wundheilung und damit das Therapieversagen mit der Zeit minimieren sollte $[49,52]$.

Konnte der IOD nicht ausreichend gesenkt werden, so sollte eine spätere TE mit den gleichen Erfolgsaussichten möglich sein wie vor der Phako-ELT, da weder durch die Clear-cornea-Kataraktoperation noch durch die ELT die Konjunktiva verletzt und eine Vernarbung induziert wurde.

In unserer Untersuchung zeigte sich eine IOD-Reduktion um $8,79 \mathrm{mmHg}$ $(-37,70 \%, \mathrm{p}<0,001)$ mit einer gleichzeitigen Reduktion der AGD um 0,79 Medikamente $(\mathrm{p}=0,017)$.

Es ist bekannt, dass die Kataraktoperation selbst den IOD senkt $[10,17,24,38$, $39,40]$. Dooley et al. [10] sowie Kim et al. [24] fanden eine Reduktion des IOD um 3,2 $\mathrm{mmHg}$ bzw. 2,9 mmHg durch Kataraktoperation. Hudovernik und Pathor [17] konnten lediglich eine Drucksenkung von 1,4 mmHg 1 Jahr nach Kataraktoperation finden, ebenso Shingelton et al. [17], die ihre Patienten 1 Jahr nach Kataraktoperation zusätzlich in eine Glaukom- (1,11 mmHg) und eine gesunde Kontrollgruppe $(2,05 \mathrm{mmHg})$ splitteten. Shingelton et al. [39] konnten in einer späteren Arbeit zeigen, dass auch noch nach 5 Jahren in den beiden Subgruppen eine Drucksenkung von $1,8 \mathrm{mmHg}$ bzw. 1,5 mmHg gehalten werden konnte. Die Drucksenkung durch Phako-ELT $(8,79 \mathrm{mmHg})$ übertrifft die Drucksenkung durch alleinige Kataraktoperation um ein Vielfaches [29, 54]. Die Drucksenkung durch Phako-ELT ist als kumulativer Effekt aus Kataraktoperation und ELT $\mathrm{zu}$ werten.

Wir fanden eine Erfolgsrate („qualified success") von 64,3\% nach 12 Monaten. Dies ist vergleichbar mit publizierten Erfolgsraten nach TE $[23,26]$. Dies ist bemerkenswert, wenn man bedenkt, dass die Phako-ELT minimal-invasiv und nicht fistulierend ist, was einen der Hauptvorteile des Eingriffs darstellt.

Sollten nicht ausgeprägte, zirkuläre, vordere Synechien bestehen, so ist aus unseren eigenen Erfahrungen die Excimer-Laser-Trabekulotomie auch bei en-

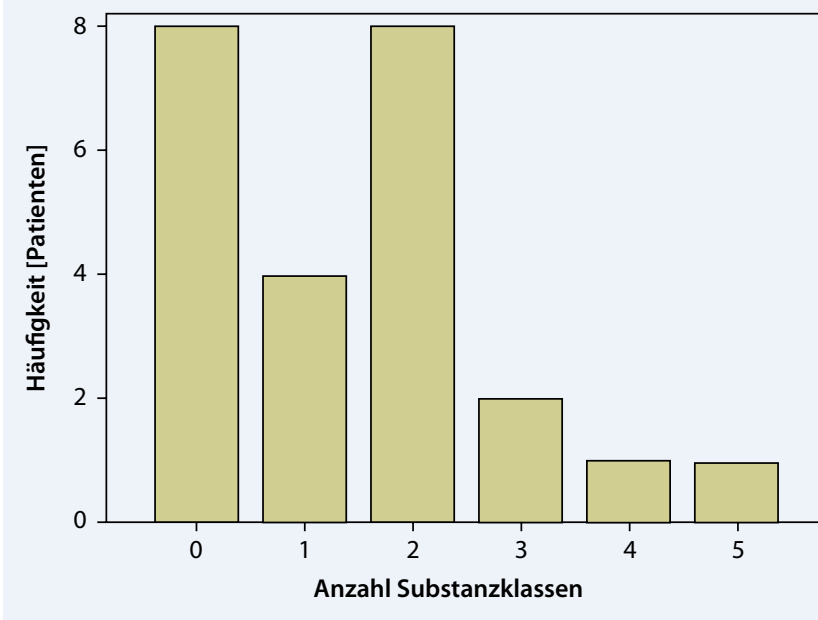

Abb. 5 - Häufigkeit der applizierten drucksenkenden Medikamente postoperativ

\section{Literatur (Auswahl)}

geren Kammerwinkeln möglich, wenn die Vorderkammer mit ausreichend Viskoelastikum vertieft wird.

$\mathrm{Zu}$ bedenken ist jedoch, dass lediglich Patienten mit einem IOD von $>21 \mathrm{mmHg}$ und $<35 \mathrm{mmHg}$ eingeschlossen wurden. Wir denken, dass die Regulation höherer Ausgangs-IOD weiterhin der TE vorbehalten sein sollte.

\section{Fazit für die Praxis}

Die kombinierte Operation von Phakoemulsifikation und Excimer-Laser-Trabekulotomie (Phako-ELT) ist für ausgesuchte Glaukompatienten mit einer Katarakt und einem moderat erhöhten IOD ohne medikamentöse Therapie oder mit einer moderaten Katarakt und einem erhöhten IOD mit medikamentöser Therapie eine gute Option, den IOD suffizient und langfristig zu senken und die lokale drucksenkende Medikation zusätzlich zu reduzieren.

\section{Korrespondenzadresse \\ M. Töteberg-Harms}

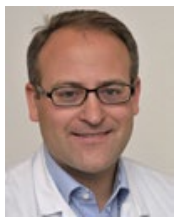

UniversitätsSpital Zürich, Augenklinik

Frauenklinikstr. 24, 8091 Zürich Schweiz

marc.toeteberg@usz.ch

Interessenkonflikt. Der korrespondierende Autor gibt an, dass kein Interessenkonflikt besteht.
1. Babighian S, Caretti L, Tavolato M et al (2010) Excimer laser trabeculotomy vs 180 degrees selective laser trabeculoplasty in primary open-angle glaucoma. A 2-year randomized, controlled trial. Eye 24:632-638

2. Babighian S, Rapizzi E, Galan A (2006) Efficacy and safety of ab interno excimer laser trabeculotomy in primary open-angle glaucoma: two years of follow-up. Ophthalmologica 220:285-290

7. Cairns JE (1968) Trabeculectomy. Preliminary report of a new method. Am J Ophthalmol 66:673679

8. Collaborative Normal-Tension Glaucoma Study Group (1998) Comparison of glaucomatous progression between untreated patients with normaltension glaucoma and patients with therapeutically reduced intraocular pressures. Am J Ophthalmol 126:487-497

9. Collaborative Normal-Tension Glaucoma Study Group (1998) The effectiveness of intraocular pressure reduction in the treatment of normal-tension glaucoma. Am J Ophthalmol 126:498-505

11. Feiner L, Piltz-Seymour Jr (2003) Collaborative initial glaucoma treatment study: a summary of results to date. Curr Opin Ophthalmol 14:106-111

15. Herdener S, Pache M (2007) Excimer laser trabeculotomy: minimally invasive glaucoma surgery. Ophthalmologe 104:730-732

16. Huang S, Yu M, Feng G et al (2001) Histopathological study of trabeculum after excimer laser trabeculectomy ab interno. Yan Ke Xue Bao 17:11-15

19. Jahn R, Lierse W, Neu W et al (1992) Macroscopic and microscopic findings after excimer laser treatment of different tissue. J Clin Laser Med Surg 10:413-418

20. Jay JL, Allan D (1989) The benefit of early trabeculectomy versus conventional management in primary open angle glaucoma relative to severity of disease. Eye 3(Pt 5):528-535

22. Kaufmann R, Hibst R (1989) Pulsed Er YAG- and $308 \mathrm{~nm}$ UV-excimer laser: an in vitro and in vivo study of skin-ablative effects. Lasers Surg Med 9:132-140

25. Migdal C, Gregory W, Hitchings R (1994) Longterm functional outcome after early surgery compared with laser and medicine in open-angle glaucoma. Ophthalmology 101:1651-1656; discussion 1657 
27. Muller-Stolzenburg N, Muller GJ (1989) Transmission of $308 \mathrm{~nm}$ excimer laser radiation for ophthalmic microsurgery-medical, technical and safety aspects. Biomed Tech (Berl) 34:131-138

28. Neuhann T, Scharrer A, Haefliger E (2001) Excimer laser trabecular ablation ab interno (ELT) in the treatment of chronic open-angle glaucoma. A pilot study. Ophthalmo Chirurgie 13:3-7

29. Pache M, Wilmsmeyer S, Funk J (2006) Laser surgery for glaucoma: excimer-laser trabeculotomy. Klin Monatsbl Augenheilkd 223:303-307

34. Quigley HA, Addicks EM, Green WR et al (1981) Optic nerve damage in human glaucoma. Il. The site of injury and susceptibility to damage. Arch Ophthalmol 99:635-649

41. Spencer WH (1972) Symposium: microsurgery of the outflow channels. Histologic evaluation of microsurgical glaucoma techniques. Trans Am Acad Ophthalmol Otolaryngol 76:389-397

42. Taliaferro K (2008) Excimer laser trabeculotomy. Ophthalmologica 222:424

43. The Agis Investigators (2000) The Advanced Glaucoma Intervention Study (AGIS): 7. The relationship between control of intraocular pressure and visual field deterioration. Am J Ophthalmol 130:429-440

48. Vogel M, Lauritzen K (1997) Selective excimer laser ablation of the trabecular meshwork. Clinical results. Ophthalmologe 94:665-667

49. Vogel M, Lauritzen K, Quentin CD (1996) Targetted ablation of the trabecular meshwork with excimer laser in primary open-angle glaucoma. Ophthalmologe 93:565-568

50. Vogel M, Scheurer G, Neu W et al (1990) Ablation of the trabecular meshwork. Klin Monatsbl Augenheilkd 197:250-253

51. Vogel MH, Schildberg $P$ (1973) Histological findings after experimental laser-trabeculo-puncture (author's transl). Klin Monatsbl Augenheilkd 163:353-358

52. Walker R, Specht H (2002) Theoretical and physical aspects of excimer laser trabeculotomy (ELT) ab interno with the AIDA laser with a wave length of $308 \mathrm{~mm}$. Biomed Tech (Berl) 47:106-110

53. Wilensky JT (1999) The role of medical therapy in the rank order of glaucoma treatment. Curr Opin Ophthalmol 10:109-111

54. Wilmsmeyer S, Philippin H, Funk J (2006) Excimer laser trabeculotomy: a new, minimally invasive procedure for patients with glaucoma. Graefes Arch Clin Exp Ophthalmol 244:670-676

\section{Das vollständige Literaturverzeichnis ...}

... finden Sie in der html-Version dieses Beitrags im Online-Archiv auf der Zeitschriftenhomepage www.DerOphthalmologe.de

\section{Möchten Sie einen Beitrag für Der Ophthalmologe einreichen?}

\section{Wir freuen uns, dass Sie unsere Zeitschrift Der Ophthalmologe mitgestalten möchten.}

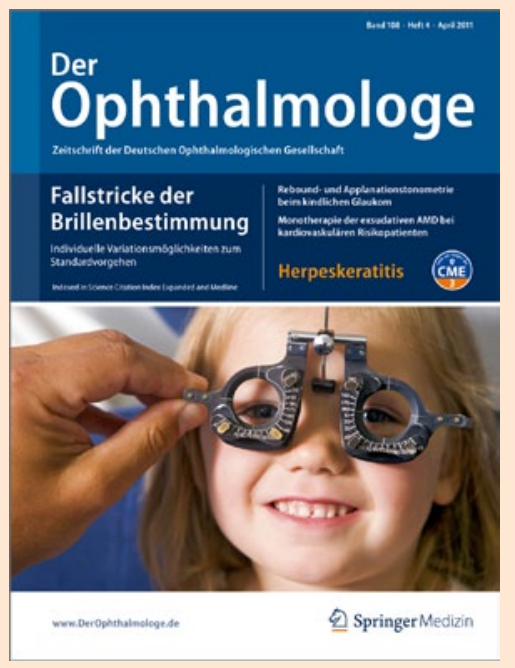

Für folgende Rubriken können

Manuskripte eingereicht werden:

- Übersichten

- Originalien

- Kasuistiken

- Bild und Fall

- Das therapeutische Prinzip

- Medizin aktuell

Um Ihnen bei der Manuskripterstellung behilflich zu sein, haben wir für unsere Autoren ausführliche Autorenleitfäden und Musterbeiträge für die verschiedenen Rubriken zusammengestellt. Diese und weitere Hinweise zur Manuskripterstellung finden Sie online unter

www.DerOphthalmologe.de/Autoren

Bitte reichen Sie Ihre Manuskripte über das Online-System „Editorial Manager" ein. Wählen Sie hierzu den Navigationspunkt "Manuskripte online einreichen"
Sollten Sie noch Fragen zur

Manuskriptgestaltung oder Einreichung haben, wenden Sie sich bitte an das Editorial Office:

Dr. Suzan Hunt

Universitäts-Augenklinik Bonn

Ernst-Abbe-Str. 2

53127 Bonn

Tel.: 02244-918491

Suzan.Hunt@ukb.uni-bonn.de

Wir freuen uns auf Ihre Beiträge!

Ihre Redaktion von Der Ophthalmologe 